\title{
Current Role and Benefits of Sentinel Lymph Node Mapping in Endometrial Cancer
}

\author{
Shrestha Sugandha, Yang Shen*, Bajracharya Prayes, Silwal Bal Mukunda \\ Department of Obstetrics and Gynecology, Zhongda Hospital Affiliated to Southeast University, Nanjing, China \\ Email: *shenyang0924@sina.cn
}

How to cite this paper: Sugandha, S. Shen, Y., Prayes, B. and Mukunda, S.B. (2020) Current Role and Benefits of Sentinel Lymph Node Mapping in Endometrial Cancer. Open Journal of Obstetrics and Gynecology, 10, 478-492.

https://doi.org/10.4236/ojog.2020.1040043

Received: March 2, 2020

Accepted: April 5, 2020

Published: April 8, 2020

Copyright ( $) 2020$ by author(s) and Scientific Research Publishing Inc. This work is licensed under the Creative Commons Attribution International License (CC BY 4.0).

http://creativecommons.org/licenses/by/4.0/

\begin{abstract}
Endometrial cancer (EC) is one of the three major malignant tumors of the female reproductive system in developed countries. In recent years, the incidence of EC ranks first in gynecological malignancy and the mainstay of treatment is Surgery. Although EC has a high prevalence, its several features associated with management are still unclear. Endometrial cancer lymph node status is the most important predictor of survival and also guides postoperative treatment planning but in gynecologic oncology, lymphadenectomy, as a part of initial surgical management remains controversial. The current introduction of sentinel lymph node mapping in EC patients represents a midway between the execution and omission of node dissection. Sentinel lymph node (SLN) mapping has emerged as an alternative staging strategy hence, the role of SLN mapping is currently being evaluated prospectively in several centers. The objective of this review is to provide an update of the emerging role of sentinel node mapping in EC. In this review, we discussed how several different techniques, type of tracers utilized and injection sites has been described and used for lymphatic mapping in EC impacting on sentinel lymph node detection rates. In addition, we also discussed another evolving field in EC staging i.e. interpretation of pathologic ultra staging of SLN, which can identify low volume metastasis for which the clinical significance and ideal management remain uncertain. Future perspective regarding EC management is also discussed in this review.
\end{abstract}

\section{Keywords}

Endometrial Cancer, Sentinel Lymph Node Mapping, Laparoscopy, Robotic Surgery, Ultrastaging

\section{Introduction of EC}

Endometrial cancer (EC) represents one of the most common gynecological ma- 
lignancies in developed countries with estimated incidence of approximately 63,000 newly diagnosed EC cases in 2018, in United States [1]. According to the data from US statistics, its incidence has increased of more than 23,000 newly diagnosed cases/year in last decade [2]. Several features of EC are fully investigated due to the high prevalence of this malignancy. Clear guidelines recommend several types of preoperative workup, surgical as well adjuvant treatments [3]. However, we should admit that few aspects of its management are yet to be clear. Specifically, data, which support the execution of retroperitoneal staging, are discordant [4]. Recent guidelines suggested that surgical procedure should include evaluation of the pelvic and para-aortic nodes [4] [5] [6].

Data on the therapeutic value of lymphadenectomy are inconsistent, thus highlighting the unmet need for this problem. Several large and well-conducted retrospective studies showed the potential benefits of lymphadenectomy in EC [7] [8]. However, two independent randomized trials emphasizing the role of lymphadenectomy in EC report that the implementation of lymphadenectomy increases patient morbidity and worsens perioperative outcome without affecting long-term outcomes [9] [10]. The ASTEC trial and Italian trial by Benedetti Panici et al. [9] include more than 1900 patients randomized to undergo hysterectomy and bilateral salpingo-oophorectomy (BSO) plus lymphadenectomy vs. hysterectomy and BSO alone. The cumulative results of these studies showed that lymphadenectomy did not improve disease-free survival (pooled hazard ratio $[\mathrm{HR}]=1.23 ; 95 \%$ confidence interval $[\mathrm{CI}]=0.96-1.58$ ) and overall survival [(pooled HR $=1.07 ; 95 \% \mathrm{CI})=0.81-1.43][11]$.

According to results of the SEPAL trial, the para-aortic lymphadenectomy has no effect on low-risk EC, but it improves the prognosis of patients with moderate-to-high-risk disease [International Federation of Obstetrics and Gynecology (FIGO)] Grade 1 and 2 tumors limited to the inner half of the myometrium with lymphovascular space invasion, FIGO grade 3 and/or non-endometrioid stage IA and IB tumor, stage IC. However, SEPAL studies also have some limitations: 1) retrospective study design, 2) low prevalence of non-endometrioid EC, and 3) relative median young age of the study population [12]. Overall, this ambiguity represents a background for changing EC treatment. In fact, the use of sentinel lymph node mapping represents the most important and innovative change in EC surgical treatment in recent decades.

In this review, our aim is to discuss: 1) the introduction of sentinel lymph node mapping in the EC, 2) how the detection rate is affected by various tracers and injection sites, and 3) the use of sentinel lymph node mapping in minimally invasive surgery, 4) Comparison of morbidity and oncology results with total lymphadenectomy; 5) Discussion of the effects of ultrastaging and the valence of micrometastases and isolated tumor cells detected in sentinel lymph nodes.

\section{Methods}

All the published series of SLN mapping in EC were searched through various database e.g. PubMed, web of science, Cochrane from 2010 to 2019 using key- 
words such as Endometrial cancer, Sentinel Lymph Node (SLN) mapping alone and in combination of SLN mapping with various tracers, injection site and surgical technique. Additionally, above-mentioned database were also searched for ultrastaging. Only relevant articles were included in this review article. The articles other than English language, letters, comments, and conference proceedings were excluded for this topic. The corresponding articles listed in the reference section were also carefully reviewed and the relevant literature was searched. Finally, through the keywords mentioned above, about 5 articles were related to our topic and were selected for this review (Figure 1).

\section{Sentinel lymph node (SLN) mapping in the EC:}

An image-guided surgical procedure in SLN mapping is being increasingly accepted in staging of apparent uterine confined endometrial cancer [13]. Gould coined discovered the term sentinel lymph node in 1960 with his observation of parotid gland cancer [14]. In 1977, Cabanas, an urologist working in Paraguay, worked as a surgical researcher at MSKCC, used lymphography and color dyes to explain the lymphatic drainage of the penis establishing the first clinical report of SLN in men with penile cancer [15]. Burke introduced the concept of SLN mapping in endometrial cancer from the MD Anderson Cancer Center in 1996 that reported 15 women undergoing open abdominal surgical mapping of lymph nodes [16]. $1 \mathrm{ml}$ blue dye was injected in the subserosal myometrium in three different uterine sites: $2 \mathrm{~cm}$ inferiorly on anterior and posterior wall of superior midpoint of the fundus. Dye uptake was observed for $10 \mathrm{~min}$ in lymphatic channels. Dye depositions into lymph nodes were seen in 10 cases (67\%) and the locations included 12 Microscopic nodal metastasis to sentinel nodes was found in 2 out of 4 women confirmed with lymphatic spread [16], but the initial results were not encouraging, and the concept has only recently gained popularity with the establishment of cervical injection techniques and the standardization of SLN mapping procedures by surgical algorithms [17] [18] [19].

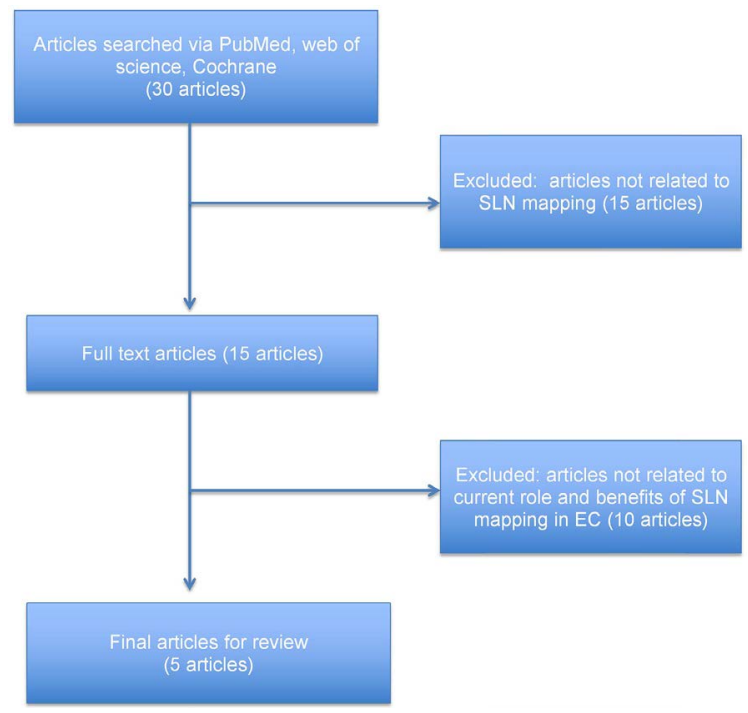

Figure 1. Flowchart showing the study inclusion. 
SLN mapping Algorithm: Reducing the incidence of surgery is an important value for SLN mapping, but the first priority when selecting this technique remains identifying all patients with lymph node metastasis. Surgeon's experience and adherence to the SLN algorithm is the key factors for successful SLN mapping procedures, which was published in 2012 and has been included in the National Comprehensive Cancer Network (NCCN) guidelines since 2014 [19] [20]. With this in mind, the MSKCC team designed an algorithm for SLN mapping for cervical and endometrial cancer [20] [21]. The algorithm requires a complete lymph node dissection on each hemi-pelvis with a failure to map SLN, all suspicious lymph nodes and peritoneal lesions and meticulous ultrastaging of SLNs. The false negative rate is in the range of $2 \%$ after applying this algorithm [21].

One of the cornerstone papers on the role of sentinel node mapping in EC, was published by the Memorial Sloan Kettering Cancer Center group [22]. In 2012, Barlin et al. [20], reported prospective data of patients having lymphatic mapping for EC. The Memorial Sloan Kettering Cancer Center (MSKCC) SLN algorithm (Figure 2) is similar to a surgical checklist, which is used to ensure standardization and reduction of the false negative rate of mapping. The algorithm considers the bilateral nature of pelvic lymph node anatomy and the possibility of severe peritoneal or retroperitoneal disease, which has recently been validated by several investigators and has good reproducibility with low false negative rates [20] [22] [23] [24]. Acceptable SLN detection rates vary among practice, but detection rates of $80 \%$ to $90 \%$ or higher are preferred [25]. Khoury-Collado and colleagues reported 115 patients with endometrial cancer treated in-between September 2005-March 2009. The overall detection rate was $85 \%$. However, in the early stages of the study (September 2005 to December 2007), 50 of the 64 cases (78\%) were identified as SLNs, with two false negative results, and in the later stages (2008 January to March 2009) out of 51 cases, 48 (94\%) SLNs were identified with no false negative results. In both time periods, the detection rate increased from $77 \%$ to $94 \%(\mathrm{p}=0.033)$, and surgeon experience (30 or more procedures) played an integral role [25] [26].

Ultimately, the National Comprehensive Cancer Network (NCCN) guidelines approved the use of sentinel lymph node mapping algorithms in the EC, thus making this method rapid and widespread in most clinical practice [19]. With the increase in surgeon experience and the corresponding increase in detection rates to $90 \%$ or higher, in addition to the reduction in false negative rates due to adherence to the SLN algorithm, SLN mapping has become a globally accepted practice for many as a standard of care.

\section{SLN mapping techniques:}

Tracer used: Various tracers have been evaluated for sentinel lymph node mapping in EC patients. They are: 1) blue dyes (methylene, isosulfan and patent blue), 2) technetium-99 radiocolloid (Tc-99m), and 3) indocyanine green (IGC).

The blue dyes are injected into the interstitial space where they bind to serum proteins and are then absorbed by the lymphatics. No requirement of dedication 


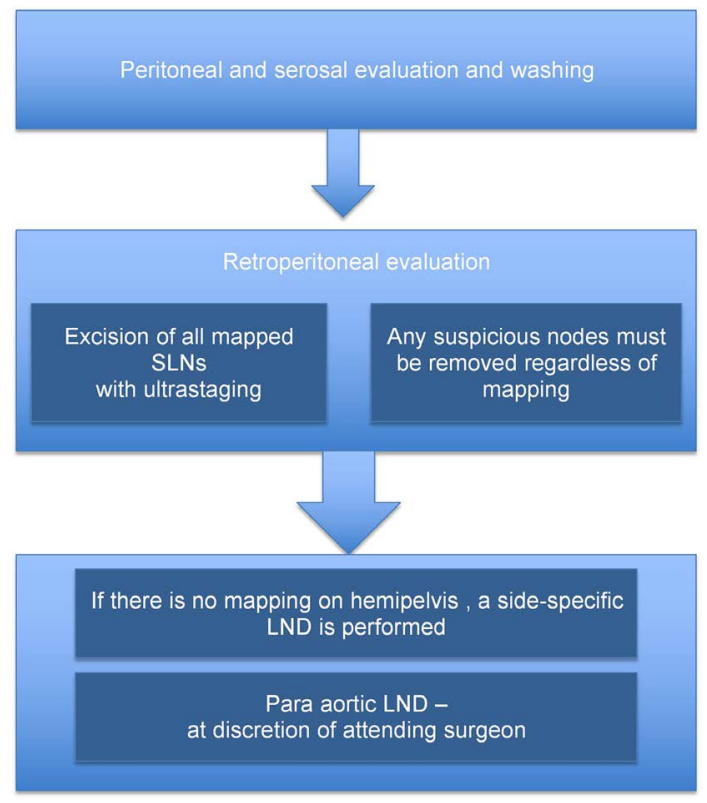

Figure 2. Sentinel lymph node (SLN) mapping algorithm. $L N D$, Lymph node dissection.

and expensive equipments are the main advantage of using blue tracers [27]. Complications of blue dye are rare, mostly consisting of allergic reactions. Montgomery and colleagues reported that in 2392 breast cancer patients, who underwent SLN mapping procedures, the incidence of allergies was $1.6 \%$ and the incidence of antihypertensive reactions was $0.5 \%$ [28].

Tc-99m is usually injected in a radiological protected setting the day before prior to surgery. The half-life of Tc- $99 \mathrm{~m}$ is about 6 hours. A gamma probe is required to detect the signal emitted by the Tc-99m and the detection is based on the audiometry signal (no color is visualized).

However, single photon emission computed tomography (SPECT-CT) execution might be helpful for obtaining more accurate information about the location of sentinel nodes. Elisei et al. [29], observed that the SPECT-CT execution is not only associated with a highest detection rate but also with bilateral mapping when compared with audiometric signal only [29]. Tc99 is not preferred because it is expensive, causes patient discomfort, and is inconvenient. Tc99 injected Intraoperative that has been reported, which is not feasible at all institutions and also introduces an added expense [30].

Indocyanine green (ICG) recently emerged as an excellent dye for SLN mapping. The $25-\mathrm{mg}$ dry powder is mixed in $20 \mathrm{~mL}$ of sterile water in the operating room, and 2 to $4 \mathrm{~mL}$ is injected directly into the cervix similarly as blue dye. The ICG consists of small particles that show fluorescence after being visualized by near-infrared light (range $700-900 \mathrm{~nm}$ ). To visualize the drainage of ICG into the lymphatics a dedicated optical system is required. The main contraindication is allergy to iodine. Recently published reports confirm that ICG with near-infrared fluorescence can result in higher overall and bilateral detection rates of SLNs 
endometrial cancer patients as compared to blue dye. In general, no matter which dye is used, successful mapping decreases with an increase in body mass index (BMI), but compared to the blue dye, the use of ICG can significantly improve. Based on these reports, most current gynecologic oncologists recommend ICG for endometrial cancer patients, especially obese patients [31] [32] [33].

The effectiveness of various tracers in terms of detection rates and bilateral mapping are compared by several studies [27] [31] [34]. Overall, these studies suggest that ICGs have higher overall and bilateral detection rates than other methods (even if they Combine [Tc-99m plus blue dye]). In addition, when ICG is used as a tracer, the detrimental effects of body mass index (BMI) in sentinel lymph node mapping are diminished [27] [31] [34]. In fact, although the accumulated data emphasizes that the increase in BMI will reduce the detection rate of sentinel lymph nodes, the fluorescence signal observed with ICG may overcome the shielding effect of adipose tissue on colorimetric signals [27] [31] [34]. Two independent studies published by Tanner et al. [35] and Eriksson et al. [31] proposed that, when using ICG, increased BMI has the detrimental effect on the detection and better visualization of sentinel lymph nodes in comparison to blue dye. The current studies agree that ICG has better safety profile than to Tc-99m (a radioactive drug) and blue dyes (reporting various adverse events, including skin necrosis) [28]. Based on this evidence, ICG is currently the preferred imaging dye at many institutions, particularly those with robotic and laparoscopic technique despite of its high cost. However, blue dye cervical injection is a "low-cost", safe and satisfactory alternative to the uterine drainage of sentinel lymph nodes.

Injection sites: Several studies have studied the best injection site for EC patients but not established yet. Three different types of SLN mapping techniques have been studied based on site of injection: 1) uterine subserosal, 2) cervical and 3) endometrial, by means of hysteroscopy. Cormier et al., compared data of 1102 and 300 patients having cervical and corporal injection, in a systematic review on sentinel node mapping, respectively [36]. The overall detection rate after cervical injection and corporal injection ranged between $62 \%-100 \%$ and $73 \%$ $95 \%$ respectively. Para-aortic node mapping was observed in $39 \%, 2 \%$, and $17 \%$ of patients having corporal, usual cervical and deeper cervical injection, respectively [36] [37].

Hysteroscopic injections are generally considered challenging. Kang and colleagues reported in a large meta-analysis that a decrease in detection rates when the cervical method was not used and also suggested avoiding "subserosal injection only" technique due to decreased sensitivity [30]. Cervical injection is simpler and wastes less time than other corporal injection methods. The multicentric sentinel node in endometrial cancer (SNEC) trial randomized patients to have cervical vs. hysteroscopic injection of ICG. Primary endpoint measures are detection rate into the para-aortic area and bilateral mapping into the pelvis, whereas, Secondary endpoint measure included operative time, surgery-related complications as well as survival outcomes [37]. Although hysteroscopic injection 
ensures more accurate visualization of tumor lymphatic drainage, cervical injection is simpler and less demanding than hysteroscopic procedure. In fact, the ease of use of the system is very essential in the actual operation and its widespread adoption.

Minimal invasive surgery: Minimally invasive surgery is increasingly used in EC staging, which has led to the growing popularity of sentinel lymph node mapping techniques. The use of innovative laparoscopic and robotic assisted system that includes software for identifying sentinel nodes represents one of the main advantages of this field [35]. The use of minimally invasive techniques (such as laparoscopy) not only cleaned up surgery, reduce postoperative morbidity and improve the quality of life of patients, but its direct vision and high magnification lens can also magnified by 10 - 20 times so that researchers can dynamically observe the drainage of tracer in regional lymph nodes and accurately distinguish the stained lymph nodes. Recently, Rossi and colleagues published the results of the FIRES trial confirming these results [38]. The FIRES trial is a multicenter, prospective cohort study that focuses 385 patients with significant stage I EC undergoing robotic-assisted surgery and had hysterectomy along with sentinel lymph node mapping followed by pelvic (with or without) para-aortic lymphadenectomy [38]. At least one sentinel lymph node mapping was observed in $86 \%$ of cases. 41 patients were identified with positive lymph nodes (among them 36 patients had at least one mapped lymph node). Nodal metastasis was identified into sentinel nodes in 35 cases (97\%) of the latter group of patients. Based on these numbers, the sensitivity of detecting lymph node-positive disease is $97.2 \%$, and the negative predictive value is $99.6 \%$ [38]. Soliman reported the overall SLN detection rate and bilateral detection rate was $89 \%$ and $58 \%$ respectively [39]. This was in line with a recent meta-analysis by Smith et al., which reported an overall detection rate and a bilateral detection rate of $81 \%$ and $50 \%$ respectively when compiling data from 55 published studies on EC SLN mapping [40].

Therefore, sentinel lymph node mapping was observed to be very effective in minimally invasive surgery hence, rapidly becoming the gold standard method for identifying sentinel lymph nodes due to the spectacular view of the laparoscope and the innovative technological system that can visualize various concentrations of the tracer.

Comparison of morbidity and oncology results with total lymphadenectomy:

The implementation of Sentinel lymph node mapping rather than full lymphadenectomy (pelvic \pm para-aortic) reduces the incidence of perioperative complications [41]. In fact, some perioperative events including lymphatic-specific complications are lymphoedema, lymphorrhea, and lymphoceles. Although the introduction of minimally invasive surgery has greatly reduced the incidence of lymphatic complications but they still appear as a major health problems for patients with lymph node dissection [42] [43]. Geppert et al. [43] 
compared the perioperative outcomes of patients undergoing hysterectomy alone vs. hysterectomy plus sentinel nodes and hysterectomy plus total lymphadenectomy. They observed, the additional average surgery time for removal of sentinel lymph nodes and full lymphadenectomy was 33 minutes and 91 minutes respectively. The prevalence of lymphedema in the legs was significantly lower after sentinel lymph node mapping as compared to full lymphadenectomy $(1.3 \%$ vs. 18.1\%; $\mathrm{p}<0.001$ ) [43]. As mentioned earlier, the FIRES test reports sentinel lymph node mapping sensitivity is higher than 95\% [38]. Recently, 2 comparative studies were conducted between two referral centers, the Mayo Clinic (Rochester, MN, USA) and the Memorial Sloan Kettering Cancer Center (New York, USA) [41] [44]. These centers conducted 2 excellent approaches in low-risk EC patients (with endometrioid histology and limited myometrial involvement). At the Memorial Sloan Kettering Cancer Center, uses a sentinel lymph node localization algorithm (described above). At the Mayo Clinic, total pelvic and para-aortic lymphadenectomy was performed in specific cases considered to have a risk of nodal metastasis (FIGO grade 3 and/or primary tumor diameter $>2 \mathrm{~cm}$ ) [44]. Increasing evidence indicated that sentinel lymph node mapping does not affect the outcomes as compared to patients with full node dissection [38] [44] [45]. Buda et al. confirmed data and reported higher prevalence of positive lymph nodes in-patient undergoing sentinel lymph node mapping as compared to conventional lymph node dissection group (16.7\% and $7.3 \%$ of patients with positive pelvic lymph nodes, in sentinel node mapping and lymphadenectomy groups respectively; $p=0.002$ ) [46]. The accuracy of sentinel lymph node mapping has been evaluated by multiple studies in identifying patients with lymphatic disease [46] [47] [48] [49].

Data were collected and evaluated for more than 1100 patients; 642 (57\%) for sentinel lymph node mapping and 493 (43\%) for lymphadenectomy. Pelvic lymph nodes were removed in $93 \%$ and $58 \%$ of patients respectively $(\mathrm{p}<0.001$ ); para-aortic lymph node was removed in $14.5 \%$ and $50 \%$ of patients respectively ( $p<0.001)$. Pelvic lymph node metastases (including micrometastasis and isolated tumor cells) were found in $5.1 \%$ and $2.6 \%$ of patients, respectively ( $\mathrm{p}=$ 0.03 ), and metastasis rates in the para-aortic nodes were $0.8 \%$ and $1.0 \%$, respectively $(\mathrm{p}=0.75)$. Survival rates were similar to the 3 -year disease-free survivalrate, i.e. $94.9 \%(95 \% \mathrm{CI}=92.4$ - 97.5) and 96.8\% (95\% CI = 95.2 - 98.5), respectively [49]. A similar comparison was conducted by 2 Italian institutions [50]. The median follow-up time was shorter than 3 years, and the authors reported no difference in recurrence and mortality between the groups. However, the retrospective nature and limited follow-up periods of these studies limits the value of these findings and therefore requiring further prospective assessment.

Sentinel lymph node mapping has similar oncological outcomes than standard lymphadenectomy and patients who perform sentinel lymph node mapping may benefit from more accurate detection of lymph node metastasis. In fact, sentinel lymph node mapping improves diagnostic ability in detecting diseases residing in lymph nodes as compared to lymphadenectomy improving the detection of 
extra-uterine diseases, which may drive the choice of adjuvant therapy to improve oncological outcomes.

Ultrastaging, macrometastasis, micrometastasis and isolated tumor cells:

The proper pathological ultrastaging is an essential part when introducing the sentinel lymph node mapping process into practice. In fact, pathology protocols include $\mathrm{H} \& \mathrm{E}$ and immunohistochemical staining, as described by the American Joint Committee on Cancer (AJCC) in the breast cancer medical literature, sentinel lymph node mapping carrying diagnosis of low volume diseases: micrometastasis and isolated tumor cells [51]. All resected nodules were routinely stained with hematoxylin and eosin ( $\mathrm{H} \& \mathrm{E}$ ). If lymph node disease is detected, no further treatment is required. If the initial $\mathrm{H} \& \mathrm{E}$ staining is negative for the lymph nodes, the SLN will experience an overdue period. Using immunohistochemical (IHC) stains that perform AE1: AE3 and H \& E stains, sections $50 \mu \mathrm{m}$ apart can be obtained. Macrometastasis is defined as the tumor cells in clusters and/or lymph nodes larger than $2 \mathrm{~mm}$; the micrometastases are microscopic clusters and single cells measuring between $>0.2 \mathrm{~mm}$ to $\leq 2 \mathrm{~mm}$; the isolated tumor cells are microscopic clusters, and single cells $\leq 0.2 \mathrm{~mm}$. In various studies on this issue, the presence of small-volume lymphatic diseases ranges from $25 \%$ to $63 \%$ [38] [50] [51] [52]. Recently, Plante et al. [49] a total of 519 patients with sentinel lymph node mapping was evaluated, of which 85 were diagnosed with lymphatic disease. 43 (51\%), 11 (13\%) and 31 (36\%) patients were detected macrometastasis, micrometastasis and isolated tumor cells respectively. Plant et al. reported that the 3-year progression-free survival rate of patients with isolated tumor cells $(95.5 \%)$ was similar to that observed in node negative patients (87.6\%) and micrometastatic patients (85.5\%), but was statistically different as Compared to patients with macrometastases (58.5\%) [50]. The authors conclude that patients with isolated tumors cells should not have adjuvant therapy based solely on lymph node status, but the selection of adjuvant therapy should be adjusted according to uterine factors (e.g. histology, myometrial invasion) [50]. Preliminary data suggested that patients treated with isolated tumor cells receiving adjuvant therapy, including chemotherapy, are as effective as patients with node-negative disease. However, studies on the isolated tumor cells and long-term follow-up of untreated observational cohort tumor cells are lacking [51].

However, we must point out that the difference in adjuvant therapy may affect the results obtained by the authors, thus highlighting the need for further prospective trials of this issue. More evidence is needed due to the lack of mature data on the role of low-volume lymphatic diseases.

\section{Conclusion}

In this review, the role of sentinel node mapping in comparison to lymphadenectomy was investigated highlighting the safety and effectiveness of sentinel node mapping during endometrial cancer staging procedures that were supported by large amounts of data and the credit goes to pathological ultrastaging 
due to which sentinel lymph node mapping can detect more cases of lymphatic disease than conventional lymphadenectomy. The adoption of sentinel lymph node mapping improves the ability to identify patients with disease harboring in the lymph nodes, thus allow tailoring the adjuvant treatments. Sentinel lymph node mapping has successfully identified lymph node metastases in most women with endometrial cancer, with high sensitivity for the detection of lymphatic metastases. We must overcome the problems associated with false negative rates as the sentinel node mapping has a high diagnostic value. Paradoxically, due to the detection of isolated tumor cells, sentinel lymph node mapping may determinate overt treatment, whereas conventional staging procedure cannot detect it. Further evidence focusing on the role, benefits and pitfalls of SLN mapping are warranted. Moreover, owing to the low levels of evidence of the included studies, prospective randomized studies are needed in order to weight pros and cons of the wide spread adoption of sentinel node mapping during endometrial cancer staging procedures. Personalized medicine and molecular characterization will represent the future in the management of EC patient.

\section{Acknowledgements}

We would like to acknowledge the faculty members of the Obstetrics and Gynecology Department of the Zhongda Hospital affiliated to Southeast University for their constant support and cooperation.

\section{Conflicts of Interest}

The authors declare no conflicts of interest regarding the publication of this paper.

\section{References}

[1] Rebecca, L., Siegel, M., et al. (2018) Cancer Statistics, 2018. CA: A Cancer Journal for Clinicians, 68, 7-30. https://doi.org/10.3322/caac.21442

[2] Ahmedin, J.D., Siegel, R., Ward, E., Hao, Y.P., Xu, J.Q., Murray, T. and Thun, M.J. (2008) Cancer Statistics. CA: A Cancer Journal for Clinicians, 58, 71-96. https://doi.org/10.3322/CA.2007.0010

[3] Bogani, G., Dowdy, S.C., Cliby, W.A., Ghezzi, F., Rossetti, D., Frigerio, L. and Mariani, A. (2016) Management of Endometrial Cancer: Issues and Controversies. European Journal of Gynaecological Oncology, 37, 6-12.

[4] Bogani, G., Dowdy, S.C., Cliby, W.A., Ghezzi, F., Rossetti, D. and Mariani, A. (2014) Role of Pelvic and Para-Aortic Lymphadenectomy in Endometrial Cancer: Current Evidence. The Journal of Obstetrics and Gynaecology Research, 40, 301-311. https://doi.org/10.1111/jog.12344

[5] Bogani, G., Multinu, F., Dowdy, S.C., Cliby, W.A., Wilson, T.O., Gostout, B.S., Weaver, A.L., Borah, B.J., Killian, J.M., Bijlani, A., et al. (2016) Incorporating Robotic-Assisted Surgery for Endometrial Cancer Staging: Analysis of Morbidity and Costs. Gynecologic Oncology, 141, 218-224. https://doi.org/10.1016/j.ygyno.2016.02.016

[6] Colombo, N., Creutzberg, C., Amant, F., Bosse, T., Gonzalez-Martin, A., Ledermann, J., 
Marth, C., Nout, R., Querleu, D., Mirza, M.R., et al. (2016) ESMO-ESGO-ESTRO Consensus Conference on Endometrial Cancer: Diagnosis, Treatment and Follow-Up. International Journal of Gynecologic Cancer, 26, 2-30. https://doi.org/10.1097/IGC.0000000000000609

[7] Mariani, A., Dowdy, S.C., Cliby, W.A., Gostout, B.S., Jones, M.B., Wilson, T.O. and Podratz, K.C. (2008) Prospective Assessment of Lymphatic Dissemination in Endometrial Cancer: A Paradigm Shift in Surgical Staging. Gynecologic Oncology, 109, 11-18. https://doi.org/10.1016/j.ygyno.2008.01.023

[8] Fotopoulou, C., Savvatis, K., Kraetschell, R., Schefold, J.C., Lichtenegger, W. and Sehouli, J. (2010) Systematic Pelvic and Aortic Lymphadenectomy in Intermediate and High-Risk Endometrial Cancer: Lymph-Node Mapping and Identification of Predictive Factors for Lymph-Node Status. The European Journal of Obstetrics \& Gynecology and Reproductive Biology, 149, 199-203.

https://doi.org/10.1016/j.ejogrb.2009.12.021

[9] Benedetti Panici, P., Basile, S., Maneschi, F., Alberto Lissoni, A., Signorelli, M., Scambia, G., Angioli, R., Tateo, S., Mangili, G., Katsaros, D., et al. (2008) Systematic Pelvic Lymphadenectomy vs. No Lymphadenectomy in Early-Stage Endometrial Carcinoma: Randomized Clinical Trial. Journal of the National Cancer Institute, 100, 1707-1716. https://doi.org/10.1093/jnci/djn397

[10] Group, A.S., Kitchener, H., Swart, A.M., Qian, Q., Amos, C. and Parmar, M.K. (2009) Efficacy of Systematic Pelvic Lymphadenectomy in Endometrial Cancer (MRC ASTEC Trial): A Randomised Study. The Lancet, 373, 125-136. https://doi.org/10.1016/S0140-6736(08)61766-3

[11] Frost, J.A., Webster, K.E., Bryant, A. and Morrison, J. (2017) Lymphadenectomy for the Management of Endometrial Cancer. Cochrane Database of Systematic Reviews, 10, CD007585. https://doi.org/10.1002/14651858.CD007585.pub4

[12] Todo, Y., Kato, H., Kaneuchi, M., Watari, H., Takeda, M. and Sakuragi, N. (2010) Survival Effect of Para-Aortic Lymphadenectomy in Endometrial Cancer (SEPAL Study): A Retrospective Cohort Analysis. The Lancet, 375, 1165-1172. https://doi.org/10.1016/S0140-6736(09)62002-X

[13] Khoury-Collado, F., Murray, M.P., Hensley, M.L., Sonoda, Y., Alektiar, K.M., Levine, D.A., Leitao, M.M., Chi, D.S., Barakat, R.R. and Abu-Rustum, N.R. (2011) Sentinel Lymph Node Mapping for Endometrial Cancer Improves the Detection of Metastatic Disease to Regional Lymph Nodes. Gynecologic Oncology, 122, 251-254. https://doi.org/10.1016/j.ygyno.2011.04.030

[14] Gould, E.A., Winship, T., Philbin, P.H. and Kerr, H.H. (1960) Observations on a "Sentinel Node" in Cancer of the Parotid. Cancer, 13, 77-78. https://doi.org/10.1002/1097-0142(196001/02)13:1<77::AID-CNCR2820130114>3.0. CO;2-D

[15] Cabanas, R.M. (1977) An Approach for the Treatment of Penile Carcinoma. Cancer, 39, 456-466. https://doi.org/10.1002/1097-0142(197702)39:2<456::AID-CNCR2820390214>3.0.C O;2-I

[16] Burke, T.W., Levenback, C., Tornos, C., Morris, M., Wharton, J.T. and Gershenson, D.M. (1996) Intraabdominal Lymphatic Mapping to Direct Selective Pelvic and Paraaortic Lymphadenectomy in Women with High-Risk Endometrial Cancer: Results of a Pilot Study. Gynecologic Oncology, 62, 169-173. https://doi.org/10.1006/gyno.1996.0211

[17] Abu-Rustum, N.R. (2013) The Increasing Credibility of Sentinel Lymph Node Mapping in Endometrial Cancer. Annals of Surgical Oncology, 20, 353-354. 
https://doi.org/10.1245/s10434-012-2685-8

[18] Abu-Rustum, N.R. (2014) Sentinel Lymph Node Mapping for Endometrial Cancer: A Modern Approach to Surgical Staging. Journal of the National Comprehensive Cancer Network, 12, 288-297. https://doi.org/10.6004/jnccn.2014.0026

[19] Koh, W.J., Abu-Rustum, N.R., Bean, S., Bradley, K., Campos, S.M., Cho, K.R., Chon, H.S., Chu, C., Cohn, D., Crispens, M.A., et al. (2018) Uterine Neoplasms, Version 1.2018, NCCN Clinical Practice Guidelines in Oncology. Journal of the National Comprehensive Cancer Network, 16, 170-199.

https://doi.org/10.6004/jnccn.2018.0006

[20] Barlin, J.N., Khoury-Collado, F., Kim, C.H., Leitao, M.M., Chi, D.S., Sonoda, Y., Alektiar, K., DeLair, D.F., Barakat, R.R. and Abu-Rustum, N.R. (2012) The Importance of Applying a Sentinel Lymph Node Mapping Algorithm in Endometrial Cancer Staging: Beyond Removal of Blue Nodes. Gynecologic Oncology, 125, 531-535. https://doi.org/10.1016/j.ygyno.2012.02.021

[21] Cormier, B., et al. (2011) Establishing a Sentinel Lymph Node Mapping Algorithm for the Treatment of Early Cervical Cancer. Gynecologic Oncology, 122, 275-280. https://doi.org/10.1016/j.ygyno.2011.04.023

[22] Tschernichovsky, R., Diver, E.J., Schorge, J.O. and Goodman, A. (2016) The Role of Lymphadenectomy versus Sentinel Lymph Node Biopsy in Early-Stage Endometrial Cancer: A Review of the Literature. American Journal of Clinical Oncology, 39, 516-521. https://doi.org/10.1097/COC.0000000000000302

[23] Hagen, B., Valla, M., Aune, G., Ravlo, M., Abusland, A.B., Araya, E., Sundset, M. and Tingulstad, S. (2016) Indocyanine Green Fluorescence Imaging of Lymph Nodes during Robotic-Assisted Laparoscopic Operation for Endometrial Cancer. A Prospective Validation Study Using a Sentinel Lymph Node Surgical Algorithm. Gynecologic Oncology, 143, 479-483. https://doi.org/10.1016/j.ygyno.2016.10.029

[24] Ehrisman, J., Secord, A.A., Berchuck, A., Lee, P.S., Di Santo, N., Lopez-Acevedo, M., Broadwater, G., Valea, F.A. and Havrilesky, L.J. (2016) Performance of Sentinel lymph Node Biopsy in High-Risk Endometrial Cancer. Gynecologic Oncology Reports, 17, 69-71. https://doi.org/10.1016/j.gore.2016.04.002

[25] Khoury-Collado, F., et al. (2009) Improving Sentinel Lymph Node Detection Rates in Endometrial Cancer: How Many Cases Are Needed? Gynecologic Oncology, 115, 453-455. https://doi.org/10.1016/j.ygyno.2009.08.026

[26] Eitan, R., et al. (2015) Robotic Blue-Dye Sentinel Lymph Node Detection for Endometrial Cancer-Factors Predicting Successful Mapping. European Journal of Surgical Oncology, 41, 1659-1663. https://doi.org/10.1016/j.ejso.2015.09.006

[27] Papadia, A., Gasparri, M.L., Buda, A. and Mueller, M.D. (2017) Sentinel Lymph Node Mapping in Endometrial Cancer: Comparison of Fluorescence Dye with Traditional Radiocolloid and Blue. Journal of Cancer Research and Clinical Oncology, 143, 2039-2048. https://doi.org/10.1007/s00432-017-2501-8

[28] Montgomery, L.L., Thorne, A.C., Van Zee, K.J., Fey, J., Heerdt, A.S., Gemignani, M., Port, E., Petrek, J., Cody, H.S. and Borgen, P.I. (2002) Isosulfan Blue Dye Reactions during Sentinel Lymph Node Mapping for Breast Cancer. Anesthesia \& Analgesia, 95, 385-388. https://doi.org/10.1213/00000539-200208000-00026

[29] Elisei, F., Crivellaro, C., Giuliani, D., Dolci, C., De Ponti, E., Montanelli, L., La Manna, M., Guerra, L., Arosio, M., Landoni, C., et al. (2017) Sentinel-Node Mapping in Endometrial Cancer Patients: Comparing SPECT/CT, Gamma-Probe and Dye. Annals of Nuclear Medicine, 31, 93-99.

https://doi.org/10.1007/s12149-016-1137-0 
[30] Kang, S., Yoo, H.J., Hwang, J.H., Lim, M.C., Seo, S.S. and Park, S.Y. (2011) Sentinel Lymph Node Biopsy in Endometrial Cancer: Meta-Analysis of 26 Studies. Gynecologic Oncology, 123, 522-527. https://doi.org/10.1016/j.ygyno.2011.08.034

[31] Eriksson, A.G., Montovano, M., Beavis, A., Soslow, R.A., Zhou, Q., Abu-Rustum, N.R., Gardner, G.J., Zivanovic, O., Barakat, R.R., Brown, C.L., et al. (2016) Impact of Obesity on Sentinel Lymph Node Mapping in Patients with Newly Diagnosed Uterine Cancer Undergoing Robotic Surgery. Annals of Surgical Oncology, 23, 2522-2528. https://doi.org/10.1245/s10434-016-5134-2

[32] Papadia, A., et al. (2017) Sentinel Lymph Node Mapping in Patients with Stage I Endometrial Carcinoma: A Focus on Bilateral Mapping Identification by Comparing Radiotracer Tc99(m) with Blue Dye versus Indocyanine Green Fluorescent Dye. Journal of Cancer Research and Clinical Oncology, 143, 475-480. https://doi.org/10.1007/s00432-016-2297-y

[33] Sinno, A.K., Fader, A.N., Roche, K.L., Giuntoli, R.L. and Tanner, E.J. (2014) A Comparison of Colorimetric versus Fluorometric Sentinel Lymph Node Mapping during Robotic Surgery for Endometrial Cancer. Gynecologic Oncology, 134, 281-286. https://doi.org/10.1016/j.ygyno.2014.05.022

[34] Tanner, E.J., Sinno, A.K., Stone, R.L., Levinson, K.L., Long, K.C. and Fader, A.N. (2015) Factors Associated with Successful Bilateral Sentinel Lymph Node Mapping in Endometrial Cancer. Gynecologic Oncology, 138, 542-547. https://doi.org/10.1016/j.ygyno.2015.06.024

[35] Leitao, M.M., Khoury-Collado, F., Gardner, G., Sonoda, Y., Brown, C.L., Alektiar, K.M., Hensley, M.L., Soslow, R.A., Barakat, R.R. and Abu-Rustum, N.R. (2013) Impact of Incorporating an Algorithm That Utilizes Sentinel Lymph Node Mapping during Minimally Invasive Procedures on the Detection of Stage IIIC Endometrial Cancer. Gynecologic Oncology, 129, 38-41.

https://doi.org/10.1016/j.ygyno.2013.01.002

[36] Cormier, B., Rozenholc, A.T., Gotlieb, W., Plante, M., Giede, C. and Communities of Practice Group of Society of Gynecologic Oncology of C (2015) Sentinel Lymph Node Procedure in Endometrial Cancer: A Systematic Review and Proposal for Standardization of Future Research. Gynecologic Oncology, 138, 478-485. https://doi.org/10.1016/j.ygyno.2015.05.039

[37] Bogani, G., Martinelli, F., Ditto, A., Signorelli, M., Chiappa, V., Recalcati, D., Lorusso, D. and Raspagliesi, F. (2016) Sentinel Lymph Node Detection in Endometrial Cancer: Does Injection Site Make a Difference? Journal of Gynecologic Oncology, 27, e23. https://doi.org/10.3802/jgo.2016.27.e23

[38] Rossi, E.C., Kowalski, L.D., Scalici, J., Cantrell, L., Schuler, K., Hanna, R.K., Method, M., Ade, M., Ivanova, A. and Boggess, J.F. (2017) A Comparison of Sentinel Lymph Node Biopsy to Lymphadenectomy for Endometrial Cancer Staging (FIRES Trial): A Multicentre, Prospective, Cohort Study. The Lancet Oncology, 18, 384-392. https://doi.org/10.1016/S1470-2045(17)30068-2

[39] Soliman, P.T., et al. (2017) A Prospective Validation Study of Sentinel Lymph Node Mapping for High-Risk Endometrial Cancer. Gynecologic Oncology, 146, 234-239. https://doi.org/10.1016/j.ygyno.2017.05.016

[40] Bodurtha Smith, A.J., Fader, A.N. and Tanner, E.J. (2017) Sentinel Lymph Node Assessment in Endometrial Cancer: A Systematic Review and Meta-Analysis. American Journal of Obstetrics \& Gynecology, 216, 459-476e10. https://doi.org/10.1016/j.ajog.2016.11.1033

[41] Leitao, M.M. (2016) Sentinel Lymph Node Mapping in Patients with Endometrial Carcinoma: Less Can Be More. Current Obstetrics and Gynecology Reports, 5, 
279-285. https://doi.org/10.1007/s13669-016-0178-7

[42] Bogani, G., Ditto, A., Leone Roberti Maggiore, U., Lorusso, D. and Raspagliesi, F. (2017) Sentinel-Lymph-Node Mapping in Endometrial Cancer. The Lancet Oncology, 18, e234. https://doi.org/10.1016/S1470-2045(17)30247-4

[43] Geppert, B., Lonnerfors, C., Bollino, M. and Persson, J. (2018) Sentinel Lymph Node Biopsy in Endometrial Cancer-Feasibility, Safety and Lymphatic Complications. Gynecologic Oncology, 148, 491-498. https://doi.org/10.1016/j.ygyno.2017.12.017

[44] Zahl Eriksson, A.G., Ducie, J., Ali, N., McGree, M.E., Weaver, A.L., Bogani, G., Cliby, W.A., Dowdy, S.C., Bakkum-Gamez, J.N., Abu-Rustum, N.R., et al. (2016) Comparison of a Sentinel Lymph Node and a Selective Lymphadenectomy Algorithm in Patients with Endometrioid Endometrial Carcinoma and Limited Myometrial Invasion. Gynecologic Oncology, 140, 394-399.

https://doi.org/10.1016/j.ygyno.2015.12.028

[45] Ducie, J.A., Eriksson, A.G.Z., Ali, N., McGree, M.E., Weaver, A.L., Bogani, G., Cliby, W.A., Dowdy, S.C., Bakkum-Gamez, J.N., Soslow, R.A., et al. (2017) Comparison of a Sentinel Lymph Node Mapping Algorithm and Comprehensive Lymphadenectomy in the Detection of Stage IIIC Endometrial Carcinoma at Higher Risk for Nodal Disease. Gynecologic Oncology, 147, 541-548.

https://doi.org/10.1016/j.ygyno.2017.09.030

[46] Buda, A., Di Martino, G., Restaino, S., De Ponti, E., Monterossi, G., Giuliani, D., Ercoli, A., Dell'Orto, F., Dinoi, G., Grassi, T., et al. (2017) The Impact on Survival of Two Different Staging Strategies in Apparent Early Stage Endometrial Cancer Comparing Sentinel Lymph Nodes Mapping Algorithm and Selective Lymphadenectomy: An Italian Retrospective Analysis of Two Reference Centers. Gynecologic Oncology, 147, 528-534. https://doi.org/10.1016/j.ygyno.2017.09.033

[47] Kataoka, F., Susumu, N., Yamagami, W., Kuwahata, M., Takigawa, A., Nomura, H., Takeuchi, H., Nakahara, T., Kameyama, K. and Aoki, D. (2016) The Importance of Para-Aortic Lymph Nodes in Sentinel Lymph Node Mapping for Endometrial Cancer by Using Hysteroscopic Radio-Isotope Tracer Injection Combined with Subserosal Dye Injection: Prospective Study. Gynecologic Oncology, 140, 400-404.

https://doi.org/10.1016/j.ygyno.2015.12.023

[48] Ruscito, I., Gasparri, M.L., Braicu, E.I., Bellati, F., Raio, L., Sehouli, J., Mueller, M.D., Panici, P.B. and Papadia, A. (2016) Sentinel Node Mapping in Cervical and Endometrial Cancer: Indocyanine Green versus Other Conventional Dyes-A Meta-Analysis. Annals of Surgical Oncology, 23, 3749-3756. https://doi.org/10.1245/s10434-016-5236-x

[49] Holloway, R.W., Abu-Rustum, N.R., Backes, F.J., Boggess, J.F., Gotlieb, W.H., Jeffrey Lowery, W., Rossi, E.C., Tanner, E.J. and Wolsky, R.J. (2017) Sentinel Lymph Node Mapping and Staging in Endometrial Cancer: A Society of Gynecologic Oncology Literature Review with Consensus Recommendations. Gynecologic Oncolo$g y$, 146, 405-415. https://doi.org/10.1016/j.ygyno.2017.05.027

[50] Plante, M., Stanleigh, J., Renaud, M.C., Sebastianelli, A., Grondin, K. and Gregoire, J. (2017) Isolated Tumor Cells Identified by Sentinel Lymph Node Mapping in Endometrial Cancer: Does Adjuvant Treatment Matter? Gynecologic Oncology, 146, 240-246. https://doi.org/10.1016/j.ygyno.2017.05.024

[51] St Clair, C.M., Eriksson, A.G., Ducie, J.A., Jewell, E.L., Alektiar, K.M., Hensley, M.L., Soslow, R.A., Abu-Rustum, N.R. and Leitao, M.M. (2016) Low-Volume Lymph Node Metastasis Discovered during Sentinel Lymph Node Mapping for Endometrial Carcinoma. Annals of Surgical Oncology, 23, 1653-1659. 
https://doi.org/10.1245/s10434-015-5040-Z

[52] Ballester, M., Naoura, I., Chereau, E., Seror, J., Bats, A.S., Bricou, A. and Darai, E. (2013) Sentinel Node Biopsy Upstages Patients with Presumed Low- and Intermediate-Risk Endometrial Cancer: Results of a Multicenter Study. Annals of Surgical Oncology, 20, 407-412. https://doi.org/10.1245/s10434-012-2683-X

\section{Abbreviations}

SLN: Sentinel lymph node

EC: Endometrial cancer

BSO: Bilateral salpingo-oophorectomy

Vs.: Versus

Tc-99m: Technetium-99 radiocolloid

ICG: Indocyanine green

SPECT-CT: Single photon emission computed tomography

FIGO: International Federation of Obstetrics and Gynecology 